\title{
Trois types d'intervention du tourisme dans l'organisation de l'espace littoral*
}

Yvette Barbaza

Professeur au Centre universitaire expérimental de Vincennes. Membre d'honneur de la Société Catalane de Géographie

Le tourisme est devenu un phénomène particulièrement expressif de notre civilisation de consommation; mais le produit offert se distingue des autres produits de consommation par deux caractères particuliers:

1. Il n'est pas détruit - théoriquement du moins et dans la plupart des caspar la consommation. L'eau, le sable, le soleil, les champs de neige, l'air vif du large ou des sommets, et ces produits même de l'action humaine que sont les monuments, les sites archéologiques, les musées ou les grandes réalisations techniques contemporaines, ne sont pas entamés par la consommation touristique. (a)

2. Il doit être consommé in situ. En ce cas ce n'est pas le produit qui va vers le consommateur mais le consommateur qui va vers le produit. Ce renversement des rapports ordinaires entre production et consommation doit nécessairement s'exprimer par une intervention originale des mouvements touristiques dans l'organisation de l'espace.

\section{L'organisation de l'espace intermédiaire entre les aires de marché et de consommation}

Entre les aires de marché et de consommation touristiques, les relais urbains,

* Aquest article va ser publicat a Annales de Géographie, núm. 434, de juliol agost de 1970.- p. 446-469 
en tant que centres d'échange et de redistribution, ne sont pas nécessaires. Tout au plus les villes peuvent-elles jouer le rôle d'étape. Mais la localisation des étapes, en raison de la diversité, des aires de départ, des motivations, des moyens de locomotion utilisés..., n’est absolument pas «déterminée». Elles peuvent s'échelonner tout au long des grands itinéraires, sans égard aux fonctions ou à la taille des villes et souvent même en dehors d'elles, si elles ne présentent pas un intérêt touristique particulier. Il en résulte un émiettement, ou tout au moins un échelonnement sans rythme précis, des installations d'accueil et des services touristiques sur les routes les plus frequentées. Et ceci d'autant plus que les préoccupations de moindre coût et de rapidité des transports sont généralement moins contraignantes pour les touristes que celles de l'agrément du voyage. D'où une plus grande liberté dans le choix des itinéraires et des étapes sur ces itinéraires. Il est vrai cependant que la masse des touristes est généralement pressée d'arriver au lieu des vacances. De sorte que la circulation touristique, même lorsqu'elle est diffuse à l'origine, se concentre assez rapidement sur quelques itinéraires privilégiés: routes du soleil, de l'eau ou de la neige, les premières ayant un avantage considérable en ce qui concerne le volume des flux et leur concentration dans le temps. C'est sur ces grands axes privilégiés que l'influence des mouvements touristiques sur l'organisation de l'espace est particulièrement sensible. Ils imposent en effet deux types d'aménagement:

- des équipements d'accueil et de service au long des routes et des villes traversées: stations -services, restaurants, hôtels, motels, campings, centres commerciaux... C'est l'expression évidente et banale de la réaction spontanée de l'offre à la demande;

- des équipements assurant la fluidité de la circulation, ce qui implique:

a) en premier lieu, une politique routière et autoroutière qui n'obéit pas aux impératifs habituels, essentiellement économiques, de la politique des transports. L'emprise du tourisme sur cette politique s'exprime clairement, par exemple, dans la programmation de l'autoroute Nice-Perpignan, la seule qui, en France, échappe totalement à la tendance centralisatrice;

b) en second lieu, un aménagement des traversées urbaines. Les interminables files d'attente à l'entrée de Béziers ou de Narbonne, pendant les mois d'été, ont acquis une notoriété qui dépasse largement nos frontières. La solution relativement simple des déviations se heurte souvent à l'opposition des populations concernées, qui redoutent l'atrophie des villes par détournement des flux qui les irriguaient. D'où la nécessité d'aménagements de la circulation toujours difficiles et d'une efficacité limitée.

Les flux touristiques s'écoulent sur ces axes privilégiés indépendamment de la structure des réseaux urbains, sur un seul plan horizontal, indifférents à la hiérarchie des villes traversées. Leur régime est éminemment irrégulier, avec d'énormes crues correspondant aux grands départs et aux grands retours, et, dans l'intervalle, un médiocre débit. Cette irrégularité et ce manque de sélectivité expliquent que l'action des mouvements touristiques sur l'organisation 
de l'espace intermédiaire entre aires de départ et d'accueil soit, beaucoup moins structurante que celle des mouvements des autres produits de consommation qui, par leurs exigences en matière d'échange et de redistribution, ont fixé l'emplacement des marchés, devenus eux-mêmes les principaux points d'appui des réseaux urbains. On peut même se demander si les mouvements touristiques n'ont pas, au contraire, une action perturbatrice, dans la mesure où ils introduisent, dans l'organisation préalable de le l'espace, un élément discordant. Ils ne s'intègrent pas, ou s'intègrent mal, aux circuits qui structurent l'espace régional. Ils se superposent aux flux régionaux réguliers et constants. Ils les débordent et les perturbent en créant des discontinuités dans le rythme de la circulation et dans la demande de services et de produits de consommation.

De sorte qu'en définitive le bilan de l'action des mouvements touristiques sur l'organisation de l'espace intermédiaire entre aires de départ et d'accueil est rarement positif.

\section{L'organisation de l'espace dans les régions d'accueil}

En revanche, au niveau des régions le tourisme devient le facteur essentiel de l'organisation de l'espace, et c'est là qu'il convient de rechercher et de définir une typologie. Comme cette recherche concerne un phénomène récent, à peine ébauché à l'échelle de l'ensemble des phénomènes humains inscrits dans l'espace, on ne peut encore prétendre en prendre une vue d'en semble. Mais on peut procéder par approches successives. C'est pourquoi nous nous bornerons à l'étude de trois types d'intervention du tourisme dans l'organisation de l'espace littoral.

Nos conclusions seront d'ailleurs largement susceptibles d'extrapolation aux autres catégories d'espaces touristiques.

Parmi les critères sur lesquels pourrait se fonder une typologie des implantations touristiques, nous retiendrons:

- le degré d'occupation de l'espace, et la plus ou moins grande vitalité et diversité des activités, antérieurement à l'intervention du tourisme:

- le caractère spontané ou volontaire des équipements:

- le caractère ponctuel ou continu des implantations. ${ }^{1}$

Lorsqu'un mouvement touristique se déclenche spontanément en faveur d'une région, l'équipement de cette région répond et s'ajuste à la demande. Si celle-ci croit très rapidement, l'équipement est hâtif, désordonné et risque de compromettre le potentiel touristique de la région. Il importe en ce cas de le contrôler et éventuellement de le freiner.

1. Nous négligeons, dans cette première approche, les nombreux critères ayant valeur moins générale, tels que : caractéristiques du milieu (climat, haute ou moyenne montagne, nature des côtes, sableuses ou accidentées), origine et niveau social de la clientèle, nombre et durée des saisons... critères qui pourront être ultérieurement intégrés dans une typologie plus élaborée. 
Si un mouvement spontané ne se produit pas en faveur d'une région dont le potentiel touristique parait cependant intéressant, on peut essayer de le provoquer:

- soit, en créant ex nibilo des stations nouvelles, indépendantes, plus ou moins isolées. On aboutit en ce cas à un aménagement ponctuel;

- soit en aménageant volontairement un ensemble touristique, intégrant des noyaux de peuplement ancien et des stations nouvelles, solidaires les uns des autres, dans un espace continu.

Ces trois modes d'intervention déterminent trois types originaux d'organisation de l'espace. Nous en étudierons trois exemples sur le littoral de la Méditerranée et de la mer Noire.

\section{$1^{\text {er }}$ type: Aires littorales atteintes par un mouvement touristique spontané: Costa Brava-Côte d'Azur}

L'une et l'autre ont attiré spontanément les touristes.

Nous ne rappelons que pour mémoire le cas de la Côte d'Azur où l'intervention du tourisme s'est produite, comme il est bien connu, en deux temps.

Ĺa concentration urbaine dans quelques stations privilégiées et l'occupation des pentes ensoleillées représentent la projection spatiale de la première vague de tourisme aristocratique hivernal. Elle a considérablement augmenté le volume des flux - hommes et capitaux-, irriguant l'espace littoral, et non moins reculé les limites des aires de départ de ces flux, intégrant ainsi la Côte d'Azur dans des circuits amples et puissants dont l'effet d'entrainement sur l'arrièrepays a été limité, mais qui ont considérablement enrichi le littoral et déclenché un processus de croissance urbaine.

L'impact du tourisme estival de masse, beaucoup plus brutal, a provoqué, surtout à partir de 1945-1950, la descente de l'habitat vers la plage et l'occupation linéaire de l'espace littoral interurbain. Les structures héritées de la première époque étant inadaptées à un phénomène de masse, il en est résulté un bouleversement du paysage, des infrastructures routières, l'apparition d'un nouveau style de constructions (grands immeubles collectifs). La fonction touristique a annexé tout l'espace littoral et regagné les pentes et même l'arrièrepays. Flux et reflux des pentes vers les plages, et réciproquement, expriment non seulement les variations de la demande touristique (soleil hivernal, bains de mer), mais aussi la pression démographique et, en particulier, l'intervention d'un nouveau facteur dont les effets sont du même ordre que ceux du tourisme, l'habitat de retraite.

Sur la Costa Brava, les manifestations du tourisme aristocratique ont été extrêmement limitées et n'ont pratiquement joué aucun rôle dans l'organisation de l'espace. Le magnifique côte de Gérone, isolée et splendide, d'accès difficile sur presque toute sa longueur, restait en dehors de tout espa- 
Figure 1

La Costa Brava avant 1950

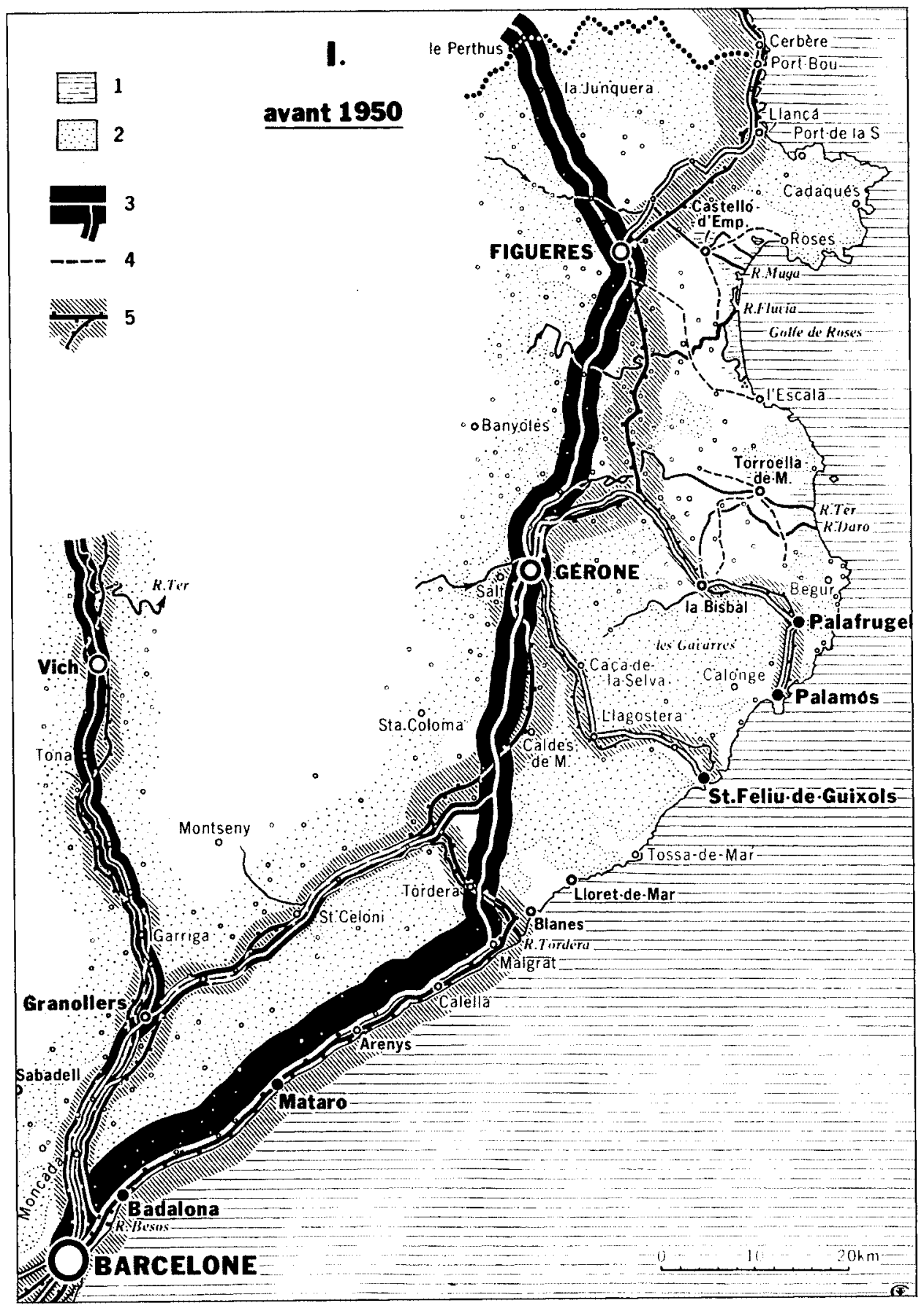

1. Zones marécageuses. 2. Zones supérieures à $100 \mathrm{~m}$. 3. Importance du flux routier. 4. Principaux courants de relation secondaires. 5. Importance du flux ferroviaire. 
ce dominé par une grande ville (fig. 1). Alors que, grâce à l'industrie du liège, des flux d'échanges et de capitaux la reliaient aux plus lointains rivages, ses relations avec Gérone et Barcelone restaient médiocres. Entre ses trois secteurs —-secteur pyrénéen, dont les petits ports désertés mouraient lentement, secteur ampurdanais, aux côtes inhospitalières (rigides falaises calcaires, longs cordons de sable), secteur méridional, encore animé par l'industrie du liège malgré les crises qui l'avaient durement éprouvée- le seul lien avait toujours été le cabotage. Celui-ci disparu, le littoral se trouvait, plus divisé, moins «organise» à l'aube du xxe siècle qu'il n'avait jamais été, lorsqu'il fut atteint par la première vague du tourisme de masse international, immédiatement après la seconde guerre mondiale. Le flot touristique grossit spontanément avec une prodigieuse rapidité. Le pays n'était absolument pas préparé à le recevoir. Les touristes précédaient l'équipement. La réponse de l'offre a été immédiate. Une véritable course à l'équipement a tenté de rattraper une demande galopante. Un vertige de constructions, avec son inévitable cortège de spéculations foncières, a gagné tout le littoral.

Pareille situation comportait deux dangers:

- danger d'un équipement anarchique qui, tôt ou tard, détruirait les magnifiques paysages de la vieille côte de Gérone désormais mondialement connue sous le nom de Costa Brava;

- danger d'un «emballement» de l'offre qui risquait de ne pas rester ajustée a la demande.

La Costa Brava n'a su éviter ni l'un ni l'autre, malgré la rapide élaboration d'un plan Costa Brava fixant des normes et imposant des règles qui n'ont guère été respectées. De sorte que le phénomène de dégradation des paysages - le plus souvent irréversible et devenu, hélas, trop banal- ne l'a pas épargnée, malgré des mises en garde répétées.

Cette irruption massive et brutale du tourisme devait nécessairement mettre en cause l'organisation antérieure de l'espace.

1) Son premier effet a été de modifier profondément les conditions de l'habitat.

D'une part, les structures de l'habitat ancien ont été affectées non seulement par la mise en place des équipements d'accueil, sportif, récréatif... mais encore par la conversion d'éléments anciens (usines de liège par exemple, conserveries de poisson ou antiques «masias» transformées en hôtels, restaurants, bars, «boîtes de nuit»... ), mais plus généralement par la rénovation et la modernisation des habitations, des infrastructures de transport urbain, des services publics...

D'autre part, un habitat linéaire continu tend à occuper tout l'espace littoral interurbain. A la progression banale de l'habitat en antennes de part et d'autre des villes s'ajoute ici une forme originale d' «urbanisation». On désigne ainsi 
l'ensemble des opérations (foncières, parcellation, lotissements, création d'infrastructures, constructions ... ) qui aboutissent à la création d'un petit complexe touristique comprenant quelques résidences individuelles ou collectives, un ou deux hôtels, des équipements sportifs, récréatifs, commerciaux... Ces «urbanisations» — dont quelques-unes seront peut-être l'amorce de nouvelles stations- servent pour l'instant de relais aux flux qui s'organisent sur l'ensemble du littoral ou qui y aboutissent.

2) En effet -et c'est la un des aspects essentiels du rôle du tourisme dans l'organisation de l'espace- la trame des relations qui viennent se nouer dans les petites villes de la Costa Brava s'est considérablement densifiée et diversifiée. Sans doute l'exportation des bouchons de liège avait-elle déjà tissé un ensemble de relations très lointaines et diverses. Mais les fils en étaient extrêmement légers. Avec leur arrière-pays en tout cas, les petits ports de la côte de Gérone n'avaient que des liens très médiocres. Les flux longitudinaux, entre la France et Barcelone, s'écoulaient directement du Perthus à la capitale catalane par Gérone et la dépression littorale de la Selva, tandis que les flux transversaux, court-circuités par cet axe majeur, étaient également détournés vers Barcelone. D'où l'isolement et la faiblesse économique des petits ports de la Costa Brava.

Le tourisme a profondément bouleversé cette situation. Les très lointaines relations n'ont guère été affectées (elles restent indépendantes du tourisme). En revanche, les relations avec l'arrière-pays, le reste de l'Espagne et les nations voisines se sont considérablement amplifiées. Des flux énormes de personnes (touristes et main-d'œuvre), de produits, de capitaux (investissements et dépenses des touristes) se sont brutalement déversés sur la Costa Brava, ont rompu son isolement, et imposé la modernisation des infrastructures de transport et la création de l'aéroport de Gérone-Costa Brava. Les flux qui traversaient la province de Gérone et se dirigeaient directement vers Barcelone sont maintenant, en grande partie, arrêtés et détournés vers le littoral. La cote de Gérone est désenclavée et intégrée à l'ensemble régional (fig. 2).

3) Elle doit enfin au tourisme une unité qu'elle n'avait jamais connue, même au temps où le cabotage créait un lien vivant entre ses petits ports. Le fait que tout le littoral, de Cerbère à Blanes, se place maintenant sous l'unique étiquette Costa Brava est significatif (naguère, on distinguait les «marines» du Haut et du Bas-Ampurdan et celle de la Selva). Sans doute l'ensemble des agglomérations littorales n'est-il pas encore nettement hiérarchisé malgré une certaine concentration des services qui incite Saint-Feliu-deGuíxols —et secondairement Roses— à revendiquer le titre de "capitale touristique» de la Costa Brava. Mais le tourisme introduit un puissant élément de solidarité et prépare l'organisation d'un espace véritablement fonctionnel, l'espace touristique.

Mais, parce que le tourisme est intervenu dans un milieu densément occupé, fortement et diversement humanisé, parce qu'il s'y est intégré sans en détrui- 
Figure 2

La Costa Brava en 1964

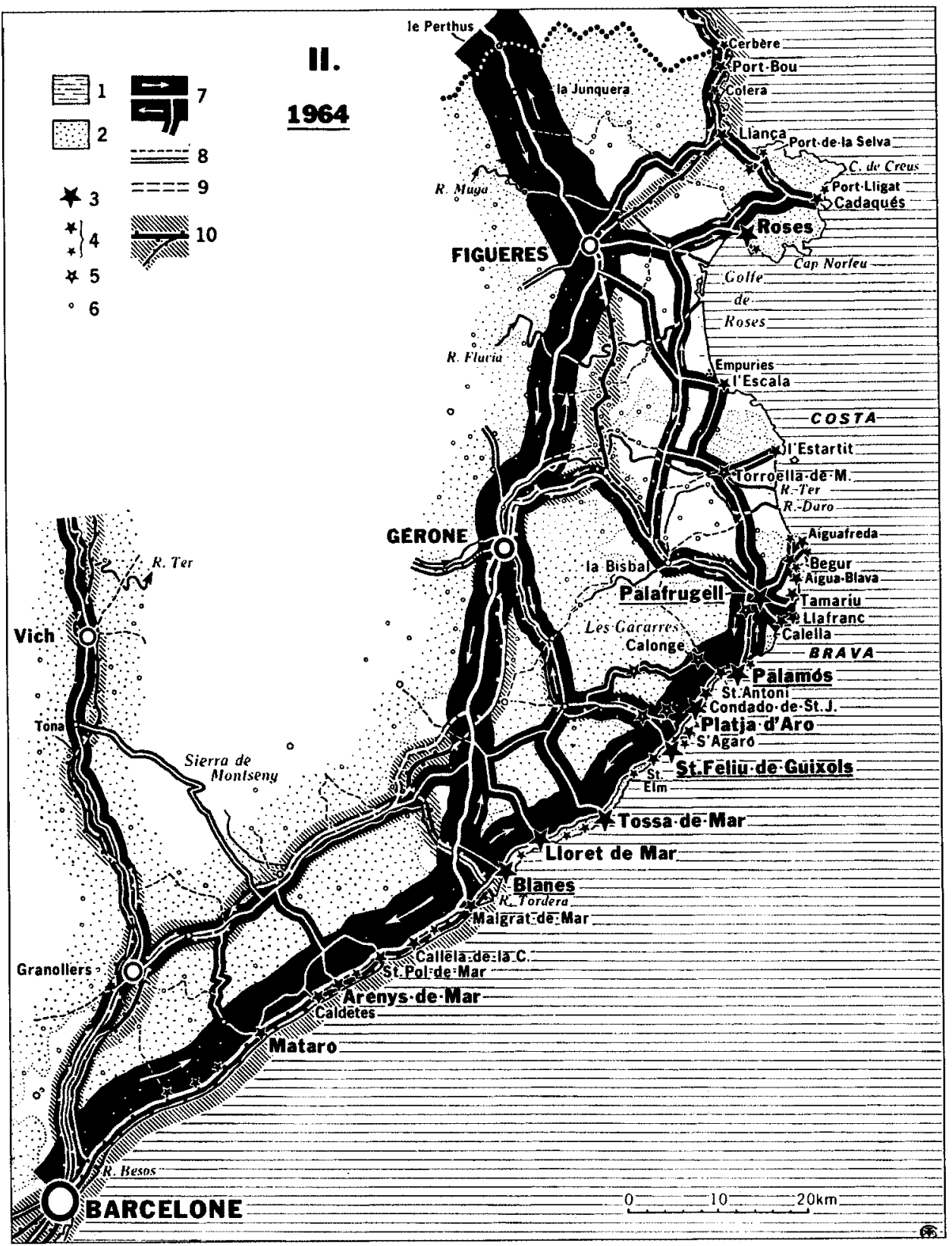

1. Zones marécageuses. 2. Zones supérieures à $100 \mathrm{~m}$. 3. Stations touristiques de premier ordre (quelques villes d'activité complexes, devenues centres touristiques, sont soulignées d'un trait). 4. Autres centres touristiques. 5. Villages agricoles touchés par le tourisme. 6. Principaux villages. 7. Importance et directions du flux touristique routier $(4,4 \mathrm{~mm}=1$ milion de voyageurs). 8. Routes secondaires. 9. Projet d'autoroute. 10. Importance du flux touristique ferroviaire $(4,4 \mathrm{~mm}=1$ milion de voyageurs). 
re l'originalité profonde, parce que —malgré les inévitables transferts de maind'œuvre- la pêche, l'industrie, l'agriculture ${ }^{2}$ y restent actives, cet espace touristique est vivant et divers. Il reste vrai. Le touriste y rencontre des traditions et des groupes sociaux authentiquement régionaux et non un folklore, apprêté et sclérosé. Il s'enrichit à ces contacts et des séjours répétés n'épuisent pas sa curiosité. Sans doute, le tourisme impose-t-il un rythme de vie saisonnier; mais il n'y a pas, en hiver, de rupture totale - sauf dans des cas très limités. La vie continue partout, simplement ralentie, retrouvant, en fin de compte, un rythme millénaire. (b)

La Costa Brava fournit donc un exemple d'espace littoral profondément humanisé, mais jusqu'ici isolé, mal structuré et manquant d'unité, auquel le tourisme a apporté, avec le désenclavement par rapport à l'arrière-pays, une unité et une organisation fonctionnelles, sans pour autant détruire sa personnalité.

\section{$2^{\mathrm{e}}$ type: «Stations» touristiques résultant d'un aménagement volontaire et ponctuel: le littoral roumain et bulgare}

Du delta du Danube à la frontière turque, le littoral de la mer Noire comprend les secteurs suivants (fig. 3):

- du cap Midia à Constantza, une cote basse, sableuse, bordée de vastes lagunes (ghiols) plus ou moins isolées de la mer par des cordons littoraux;

- à partir de Constantza, la mer taille dans le limon qui recouvre les calcaires sarmatiens (les falaises de 20 à $30 \mathrm{~m}$, qui se prolongent en territoire bulgare jusqu'à Chabla. Au-delà, les falaises sont taillées dans le calcaire gréseux du Sarmatien. Dans leurs inflexions se logent, quelques jolies plages. ${ }^{3}$

A partir de Baltchik, le liséré des plages devient, continu et s'élargit jusqu’à Varna.

- Entre Varna et Nessebar, l'extrémité orientale de la Stara Planina (Balkans) projette de lourds promontoires densément boisés, au sud desquels s'allongent les très belles plages de la Cote du Soleil (Slânčev Brjag) et de Nessebar.

- Au sud de Nessebar et surtout à partir de Bourgas, la côte est plus découpée dans les sombres roches volcaniques de la Strandja Planina, et comporte de nombreuses plages de sable doux et fin, souvent modelé en dunes, longues et belles jusqu'à Primorsko, plus petites au-delà. Au total, $100 \mathrm{~km}$ de côtes en Roumanie (en plus des 435.000 ha du delta du Danube), $378 \mathrm{~km}$ en Bulgarie (700.000 $\mathrm{m}^{2}$ de plages).

2. La pêche se maintient particulièrement active à Palamós et Roses; l'industrie du liège décline pour des raisons indépendantes du tourisme. Certaines formes d'artisanat progressent. L'agriculture se spécialise et se modernise rapidement en Ampurdan. Les activités traditionnelles ne déclinent que dans les secteurs où elles étaient, dès avant l'intervention du tourisme et du fait de la structure des entreprise, ou des explotations, frappées de marginalisme. Cf. Yv. BarbazA, Le Paysage humain de la Costa Brava, Paris, A. Colin, 1966.

3. C'est dans l'une d'elles, la baie des Oiseaux, que le c.E.T. a choisi d'installer son (village) de Roussalka. 
Figure 3

La côte bulgare et roumaine

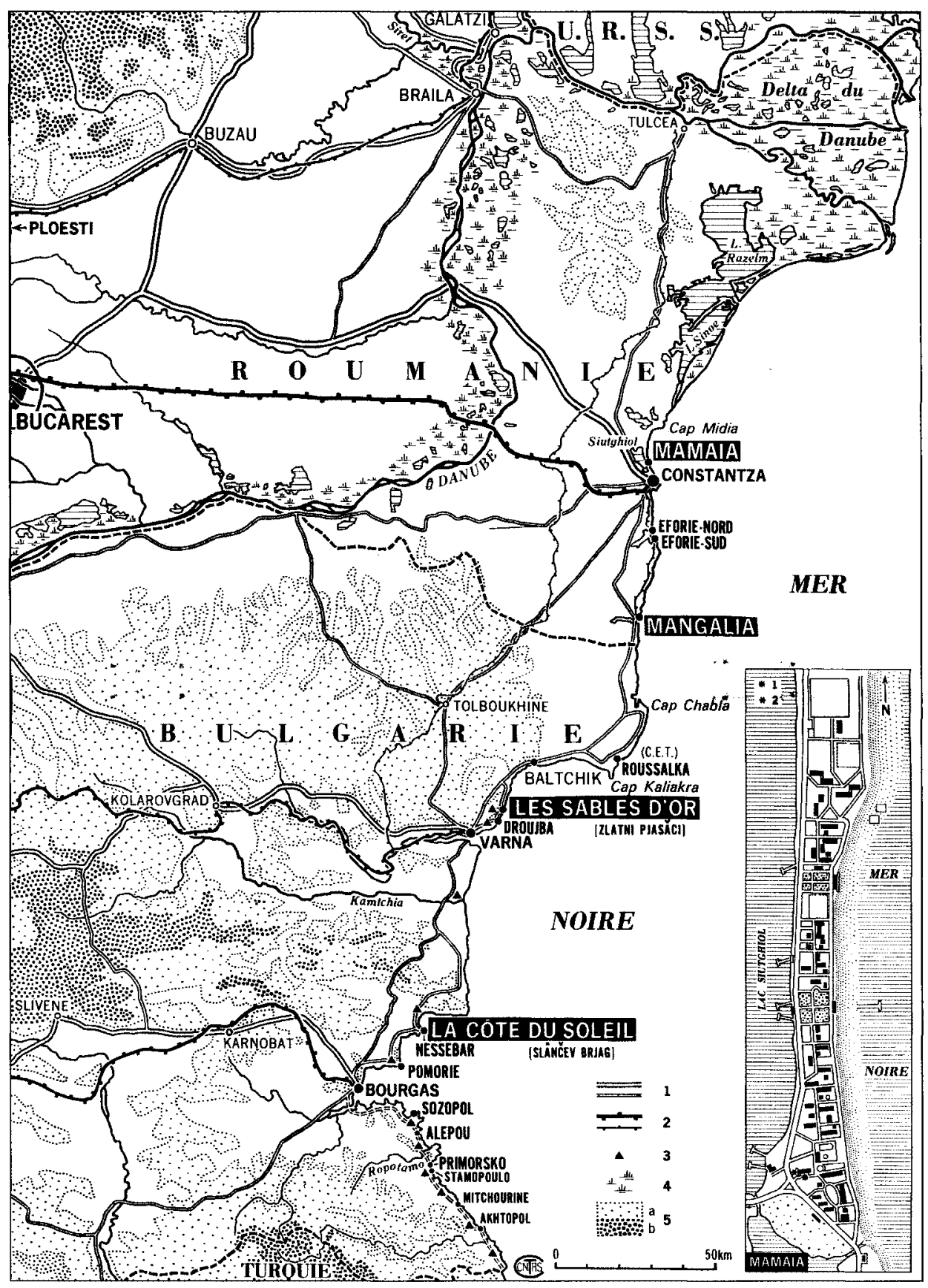

1. Routes. 2. Voies ferrées. 3. Terrain de camping. 4. Zone marécageuse. 5. Zone montagneuse: a) de 200 à $500 \mathrm{~m}$; b) au-dessus de $500 \mathrm{~m}$. Plan de Mamaia: 1. Agence de voyages des chemins de fer roumains. 2. Agence de l'office national de tourisme. 
Jusqu'à la dernière guerre mondiale, le tourisme sur le littoral bulgare se réduisait à quelques manifestations isolées et négligeables. En Roumanie, quelques résidences d'été étaient apparues à Eforie-Sud vers 1899-1900 et à Eforie-Nord vers 1910, et vers 1919 à Mamaia; un hôtel y avait été construit et la famille royale y possédait une résidence d'été, tandis que la reine Marie avait choisi Baltchik (aujourd'hui en territoire bulgare) pour y faire édifier un palais entouré de jardins magnifiques. Ces diverses manifestations, très limitées, n’avaient encore en 1940 joué aucun rôle dans l'organisation de l'espace littoral. A coté des trois grands ports maritimes de Constantza, Varna et Bourgas, commandant chacun un large hinterland, on ne trouvait que quelques petits ports de pêcheurs, plus nombreux en Bulgarie, où la cote est plus accidentée qu’en Roumanie. Contrairement à la Costa Brava, ce secteur littoral de la mer Noire n'était pas enclavé, mais l'occupation humaine était assez légère en dehors des villes et laissait de très vastes espaces disponibles pour un éventuel aménagement.

Après la seconde guerre mondiale, les préoccupations sociales des gouvernements des Républiques Populaires, leur politique de promotion humaine et leur souci de se procurer des devises devaient nécessairement attirer leur attention sur les avantages que présenterait le développement du tourisme. La première entreprise d'État pour organiser les prestations du tourisme international a été créée en Bulgarie en 1948: mais c'est surtout a partir de 1956-1957 qu'a été amorcé, dans les deux pays, un processus d'équipement touristique auquel le régime socialiste conférait des caractères originaux.

La propriété collective du sol et le rôle de l'État dans le financement des équipements levaient la plupart des contraintes humaines et financières et permettaient l'édification rapide de grands complexes touristiques relevant d'une seule entreprise et groupant 15.000 à 25.000 lits d'hôtels et tous les équipements complémentaires. Libérés des problèmes fonciers et de rentabilité à court terme, les architectes auxquels a été confiée la réalisation de ces complexes ont pu concevoir des aménagements concertés, rigoureusement planifiés.

Le processus d'équipement touristique a donc été ici exactement l'inverse de celui qui joue normalement dans un système de libre entreprise et en particulier de celui qui a joué sur la Costa Brava. La décision de créer les équipements a précédé la venue des touristes. L'expansion touristique n’a pas été ici la conséquence mais le mobile du comportement économique. Après de solides études, de marché et en fonction des possibilités d'accueil des plages dûment calculées, le gouvernement s'est fixé un objectif et «a mis en œuvre les moyens harmonisés d'atteindre cet objectif» (G. Labeau).

Ainsi ont été créées les stations, de Droujba, Les Sables d'Or (Zlatni Pjasăci), La Côte du Soleil (Slânčev Brjag) en Bulgarie, et celles de Mamaia, EforieNord et Sud, Mangalia en Roumanie. Dans tous les cas, il s'agit de "stations" créées ex nibilo, sur des territoires pratiquement vierges.

A Droujba (à $9 \mathrm{~km}$ au nord de Varna), les travaux ont commencé en 1948. La station disposait en 1968 de 18 hôtels, d'une capacité de 2.542 lits et de bungalows (144 lits) dispersés dans des jardins. 
Zlatni Pjasăci a été implantée au nord de Droujba, dans une magnifique forêt ${ }^{4}$ bordant étroitement une plage de $3,5 \mathrm{~km}$ de longueur sur 50 à $100 \mathrm{~m}$ de largeur. Les travaux ont commencé en 1956-1957. En 1968, la station avait 61 hôtels (11.000 lits), de nombreux bungalows (1.700 lits), 2 terrains de camping et tous les équipements complémentaires habituels: restaurants, "casino», cabarets, installations sportives...

Entre Varna et Bourgas, une troisième station: (Slânčev Brjag, (La Côte du Soleil), a été créée à partir de 1958 sur une longue plage de $8 \mathrm{~km}$. Elle avait, en 1968, 74 hôtels (15.000 lits) et de nombreux bungalows et campings.

Au sud de Bourgas, on ne trouve plus de «stations» nouvelles, mais, de très nombreux terrains de camping ont été aménagés (une vingtaine, totalisant plus de 30.000 places). ${ }^{5}$ Pourtant, la partie méridionale du littoral bulgare est très belle et dotée de petits ports de pêcheurs dont le charme séduit et attire peintres et artistes; mais ils ne possèdent encore aucun hôtel de grand confort. Des équipements importants y sont prévus (12.000 lits à Sozopol, 18.000 à Alépou, 6.000 au débouché du beau fleuve Ropotamo, 14.000 à Stamopoulo ... ).

En Roumanie, les embryons de stations d'Eforie-Nord et d'Eforie-Sud ont été les points de départ des créations d'unités touristiques de caractère international. Mais c'est à Mamaia qu'a été édifié le complexe le plus remarquable.

Entre 1959 et 1965, 34 hôtels totalisant 6.979 chambres et 15.761 lits ont été construits en même temps qu'était mis en place l'équipement complémentaire récréatif, sportif, services publics... Au total, la capacité d'accueil de Mamaia est de près de 18.000 places. L'ensemble est construit sur le lido qui sépare de la mer le lac d'eau douce Siutghiol. D'où un plan tout en longueur $(6,5 \mathrm{~km}$ sur 250 à $600 \mathrm{~m})$ mais aussi l'avantage d'un double plan d'eau (situation que nous retrouvons à Leucate-Barcarès sur le littoral languedocien). L'absence d'arbres sur le lido était un inconvénient. On a dû boiser entièrement et créer des jardins. Le résultat est remarquable.

La plus jeune des stations du littoral roumain est Mangalia-Nord, en bordure de la forêt de Comorova (elle-même résultat d'un boisement récent).

Toutes ces réalisations procèdent de la même conception de la «station» balnéaire et interviennent de la même façon dans l'organisation de l'espace. Conçues a priori, globalement, en fonction d'un objectif précis, elles forment des unités parfaitement définies, organisées et fonctionnelles. Implantées en terrain vierge, dans des sites choisis pour leurs qualités spécifiques, sans problèmes d'acquisitions foncières et de rentabilité à court terme, elles n'étaient soumises à aucune contrainte, sinon légères et purement techniques. D'où une liberté totale pour concevoir une composition urbanistique abordant ensemble tous les problèmes de répartition des volumes construits, des jardins, des allées, des places, des espaces boisés, des services publics, des équipements spor-

4. Forêt qu'il a fallu préalablement débarrasser d'une multitude de serpents en y déversant des centaines de hérissons!

5. 4.000 places pour le seul camping Perla a Primorsko. Ces campings sont particulièrement fréquentés par des Allemands. 
tifs.... en fonction du site et du paysage; le principe retenu partout étant de rapprocher le plus possible le touriste des éléments de la nature: de la pelouse, des arbres, de la plage, de la mer. «Les arbres et le soleil se donnent rendez-vous dans les chambres".

Le résultat est généralement remarquable.

L'équipement est parfaitement rationinel; le touriste trouve en même temps que le confort (à quelques détails près), la beauté, le calme, l'harmonie. Tout au plus pourrait-on regretter l'absence de véritables ports de plaisance (mais il n'y a pas encore de plaisanciers!) et l'insuffisance des équipements récréatifs. A ces légères réserves près, on doit convenir que tout a été conçu et prodigué pour la satisfaction du touriste. Tout a été calculé, prévu, mis en condition, «offert» à la consommation touristique. C'est l'espace-vacances à l'état pur.

Mais c'est précisément sur cette conception de l'espace-vacances qu'on peut formuler des réserves. Entièrement et exclusivement équipées pour le tourisme, les stations ne réservent pas la moindre surprise, ne laissent espérer la moindre découverte. L'initiative individuelle et la curiosité y sont très vite sans objet. Ce milieu artificialisé, déshumanisé, n'est en définitive qu'un beau décor; il manque de vie et de vérité; il manque de cette diversité qui est la marque d'un long passé et qui fait le charme et l'attrait des vieux pays; il manque de cette chaleur humaine qu'on ne trouve que dans un milieu social complexe, diversifié, oú les différents groupes s'enrichissent mutuellement même si, parfois, ils se heurtent. Il est vrai que des «excursions" sont non seulement possibles, mais organisées, soit vers les beaux rivages méridionaux de la Bulgarie où se maintiennent des formes de vie traditionnelles, soit vers le delta du Danube, monde immense et envoûtant, empire du silence et de la solitude, soit vers les grands ports des pays voisins: Odessa, Istambul, soit enfin a la découverte des montagnes magnifiques des Carpates, des Balkans, des Rhodopes... de leurs antiques monastères, de leurs cités historiques et de leurs villes nouvelles. Ainsi sont associées au tourisme balnéaire des formes complémentaires fondées sur l'exploitation de centres d'intérêt naturels, historiques ou techniques. Mais les liens ainsi créés sont superficiels dans la mesure où il s'agit le plus souvent de voyages organisés, payés d'avance et très rapides. Leur action d'entraînement sur les régions concernées est encore à peine sensible.

Sur le littoral en tout cas, la séparation des fonctions est totale. Les stations sont exclusivement, rigoureusement touristiques. Toutes les autres activités sont restées l'apanage des villes et villages sur lesquels, en revanche, le tourisme n'a guère de prise.

Son influence sur l'agriculture est très limitée. En Roumanie cependant, quelques fermes d'État se détournent de la culture du blé et de l'élevage traditionnel des ovins pour s'orienter vers les cultures maraîchères, les plantations de vergers de pommiers et de pêchers (Mangalia) ou de vignes (avec des cépages occidentaux comme le Chasselas doré, le Cardinal ou le Muscat).

Toutes les fonctions urbaines: administratives, tertiaires, industrielles, sont restées concentrées dans les trois grands ports: Constantza, Varna, Bourgas. 
Chacun de ces ports avait et conserve un pouvoir de commandement vigoureux sur un ample hinterland. Leur rôle dans l'organisation de l'espace littoral est renforcé par le tourisme dans la mesure où toutes les routes principales, les voies ferrées, les lignes aériennes et maritimes y aboutissent, de sorte qu'ils jouent le rôle de plaques tournantes concentrant et redistribuant les flux touristiques. ${ }^{6}$ Mais ils ne retiennent qu'une infime partie du tourisme de passage (chacun des trois ports ne dispose que d'un seul hôtel de $1^{\text {tc }}$ classe ouvert aux étrangers).

En revanche, les stations ne reçoivent les flux touristiques que par leur intermédiaire. Centres de consommation sans la moindre activité productrice, elles sont totalement tributaires de l'extérieur. Elles occupent dans les systèmes de relations régionaux et nationaux (et même internationaux) une position de terminus plutôt que de relais. Tous les courants y aboutissent sans autre contrepartie que «l'argent laissé par les touristes". Encore celui-ci est-il en majeure partie - en raison de la nature du tourisme dominant (voyages organisés) et du système économique (entreprises d'État) - directement dirigé sur Sofia ou Bucarest. Et le profit n'est répercuté sur le littoral que dans la mesure où des opérations d'équipement (infrastructure routière, équipement d'accueil ... ) sont décidées dans le cadre des plans nationaux.

La main-d'œuvre est en majeure partie (80 p. 100) recrutée dans les villages et les villes environnants parmi les femmes qui ne travaillent pas le reste de l'année ( 40 p. 100) et les étudiants (après un mois de stage). Les cadres spécialisés viennent des autres départements pendant l'été et y retournent pendant l'hiver, après le départ des derniers touristes. Alors la vie s'arrête totalement dans les stations complètement désertées.

Nous sommes donc en présence d'un type original d'intervention du tourisme dáns l'organisation de l'espace. Les systèmes de relations préexistants n’ont pas été bouleversés comme dans le cas de la Costa Brava, a la fois parce que ne se posait pas ici le problème du désenclavement et parce que le flot touristique, moins brutal et beaucoup moins puissant, a été guidé, orienté, en fonction d'un équipement précédant la demande dans le cadre d'une organisation économique de type socialiste.

6. Flux de plus en plus puissants si on en juge par les chiffres officiels donnés dans le tableau ci-dessous

$\begin{array}{lrr} & \text { Bulgarie } & \text { Roumanie } \\ 1959 & 148.025 & \\ 1960 & 200.602 & \\ 1961 & & 134.413 \\ 1964 & 808.694 & 534.133 \\ 1966 & 1.480 .667 & 916.502 \\ 1967 & 1.752 .214 & 1.201000\end{array}$

Les Républiques Populaires interviennent pour 70 à 75 p. 100 dans ces contingents.

7. On envisage cependant de maintenir une certaine activité hivernale. Trois hôtels seront chauffés à Mangalia-Nord. Pour l'instant, ils sont tous fermés en hiver. 
A chaque portion d'espace est restée affectée une fonction définie à l'échelon national; il ne saurait y avoir conflit d'utilisation.

La région reste essentiellement animée par les courants puissants, existant antérieurement et fondés sur les trois grands ports qui ont gardé leurs activités secondaires et tertiaires indépendantes du tourisme. L'impact des équipements touristiques est essentiellement ponctuel.

Les liens entre les «stations», serties dans l'espace littoral, et l'ensemble régional, sont des liens de dépendance plutôt que de solidarité contrairement au cas de la Costa Brava. Il n'y a pas de «région touristique» si on entend par-là un espace continu entièrement dominé par la fonction touristique, mais seulement des "noyaux" touristiques, reliés a leur environnement par des mouvements puissants mais discontinus dans le temps, qui s'effacent totalement en hiver et se superposent en été aux flux permanents, sans les infléchir de façon décisive. Les stations s'intègrent mal à l'espace littoral et à la vie régionale et cette situation résulte à la fois de la conception même de l'équipement touristique et du système économique qui implique une extrême centralisation des décisions, des investissements, des profits.

\section{$3^{\text {e }}$ type: Aménagement continu: type Languedoc- Roussillon}

Notre littoral du Languedoc possède les deux atouts généralement considérés comme décisifs pour attirer un tourisme de masse:

- le soleil des rivages méditerranéens,

— et d'immenses étendues de plages de sable fin: $160 \mathrm{~km}$ sans interruption, sur une profondeur qui dépasse fréquemment $100 \mathrm{à} 150 \mathrm{~m}$.

Or, en dehors des très petits ports de pêche devenus, entre les deux guerres, autant de petites stations attachées aux villes de l'ancienne Via Domitia (Le Grau-du-Roi à Nîmes, Palavas et Carnon à Montpellier, Le Grau-d'Agde à Agde et Pézenas, Valras à Béziers, Saint-Pierre et Gruissan à Narbonne, Canet et Argelès à Perpignan), des dizaines de kilomètres de plages restaient déserts pendant les mois d'été, alors que la Côte d'Azur et la Costa Brava recevaient des flux de touristes très supérieurs à la capacité d'accueil de leurs plages, et que ces mêmes flux s'écoulaient parallèlement au rivage, à quelques kilomètres à peine des petits ports qui auraient pu fournir le point de départ d'un équipement touristique.

En vérité, les causes de cette désaffection n'avaient rien de mystérieux. La côte languedocienne présentait, par rapport aux côtes provençale et catalane, trois inconvénients majeurs:

— côte à lagunes et, eaux stagnantes, elle était densément peuplée de moustiques;

- les vents du nord-nord-ouest (mistral, cers, tramontane) y sont fréquents et violents; ils soulèvent sur la plage des mitrailles de sable, et refroidissent fortement l'eau de mer au rivage;

- les longues étendues de sable sont monotones. 
Il apparaissait que, si ces trois inconvénients étaient surmontés, on disposerait ici d'un énorme potentiel touristique vers lequel pourrait être détoưrnée une partie des flux qui se dirigaient en masse vers les rivages méditerranéens.

Doit-on s'étonner que les capitaux privés n'aient pas été spontanément, tentés par l'entreprise ? On savait que la démoustication était techniquement possible. La protection contre le vent posait, et pose encore, un problème beaucoup plus difficile; les solutions envisagées: boisements et architecture spécialement étudiée, ne peuvent être que des palliatifs d'une efficacité limitée. Les uns et les autres peuvent cependant contribuer à rompre la monotonie du paysage. Mais toutes ces interventions postulent non seulement des investissements considérables, mais encore et surtout une action globale sur tout l'espace concerné. En matière de démoustication en particulier, les actions ponctuelles ne pouvaient en aucun cas prétendre à des résultats définitifs. Dans ces conditions, il est bien évident que l'œuvre à entreprendre n'était pas à la mesure des capitaux privés, même pas à celle des collectivités locales, qui, d'ailleurs, ont longtemps manqué de dynamisme. Seul l'État pouvait s'en charger dans le cadre de la politique d'aménagement du territoire. Après de longues études préalables, la décision fut prise de confier à une Mission interministérielle, créée en 1963, le soin de présenter un projet d'aménagement et d'en contrôler l'exécution.

Il s'agit donc d'un acte de géographie volontaire, qui peut être rapproché de la création des stations bulgares ou roumaines. En fait, il en differe à la fois par ses moyens et par ses objectifs.

- Par ses moyens, en raison des différences de régimes politiques et de systèmes économiques. Les contraintes foncières et les problèmes de rentabilité à court terme freinent l'entreprise languedocienne, malgré l'importance des interventions prises en compte par l'État. ${ }^{8}$ D'où la lenteur relative des rélisations.

- Par ses objectifs. D'une part, en effet, le project prévoit, non l'implantation d'une ou plusieurs stations indépendantes les unes des autres, mais l'aménagement global, en une seule opération, d'un espace continu couvrant l'ensemble du littoral languedocien et rousillonnais, et intégrant les agglomérations existantes.

D'autre part, l'aménagement du littoral doit avoir un effet d'entraînement sur toute la région.

Pour éviter la monotonies d'une urbanisation linéaire, le littoral a été divisé en 6 «unités tourisitiques» séparées par des espaces réservés, et présentant chacune des "centres l'intérêt» originaux, définis en fonction des particularités du milieu physique et de l'héritage historique de chaque secteur et de son arrière-pays immédiat (fig. 4).

8. Les infrastructures lourdes sont à la charge de l'État qui intervient aussi par des subventions diverses et des prêts à long terme. Les infrastructures légères son en partie assumées par les collectivités locales dans le cadre de Sociétés d'Économie mixte. Les équipements son confiés à des promoteurs sous la direction d'architectes agréés par la Mission. 
Figure 4

\section{Le littoral Languedoc-Roussillon}

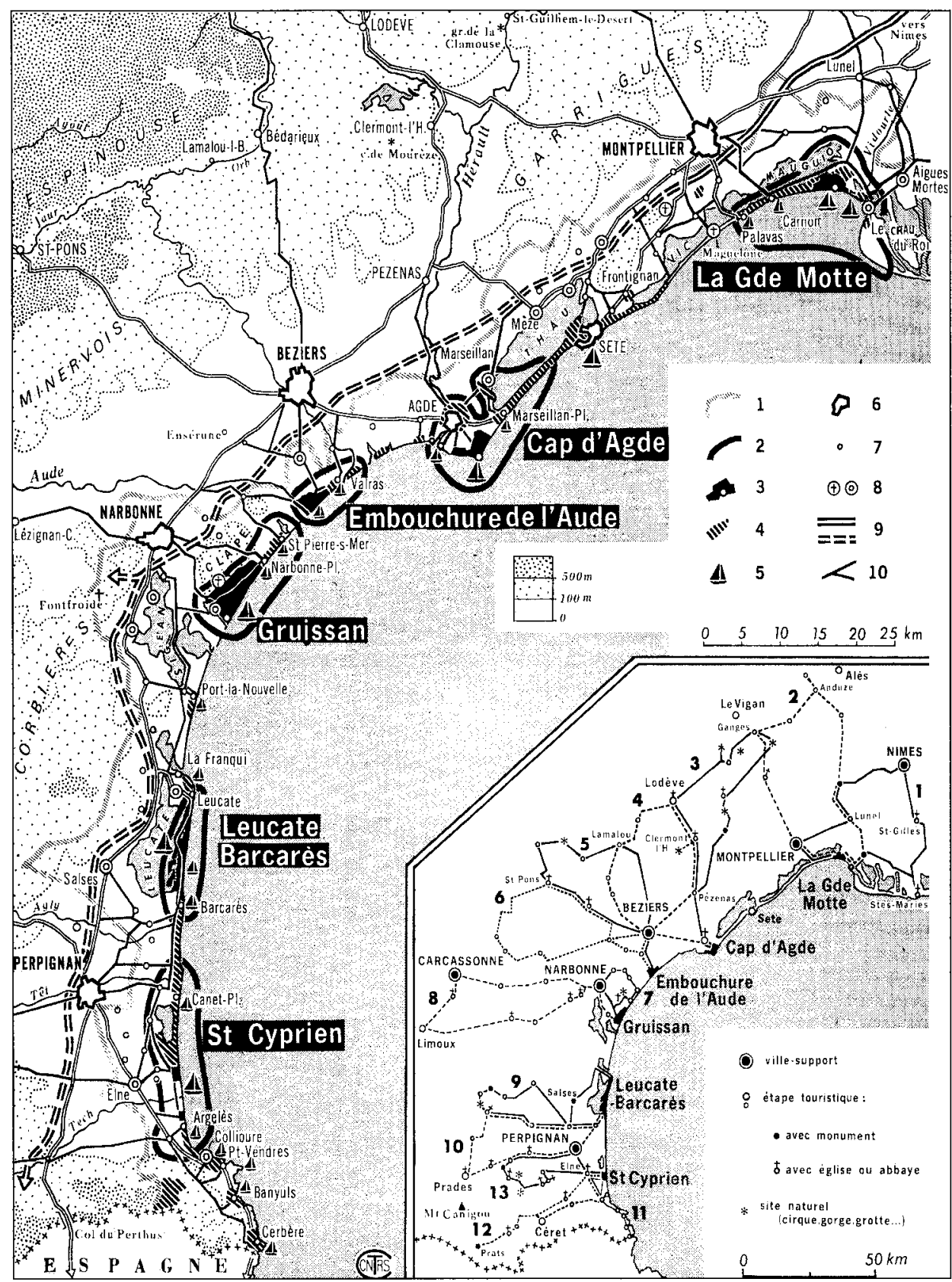

1. Limite du plan d'urbanisme d'intérêt régional. 2. Unité touristique. 3. Zone d'urbanisation préférentielle et son centre. 4. Zone d'extension de l'urbanisation. 5. Principaux ports de plaisance prévus ou construits. 6. Ville d'intérêt (ville-support). 7. Centre urbain déjà existant. 8. Agglomération ou monument à protéger. 9. Autoroute construite et en projet. 10. Routes d'intérêt regional existantes ou à aménager.

En carton, circuits touristiques au départ des centres. 
1. Du Grau-du-Roi à Palaves: trois stations existaient déjà: Le Grau-du-Roi, Carnon, Palavas. Elles son intégrées dans la première unité et une nouvelle station est créée: La Grande Motte. Lintérêt de ce secteur sera centré sur les thèmes: «Mer et Camargue» et "La Cévenne et les Camisards» (circuits 1 et 2, fig. 4).

2. Le Cap d'Agde. Une nouvelle station sera créée au Cap à Côté des anciens port du Grau-d'Agde et de la Tamaricière. Des circuits touristiques intégreront à ce secteur la vallée de l'Hérault et les Grandes Causses (circuits 3 et 4).

3. Valras et l'embouchure de l'Aude. C'est le seul secteur sans étang. Entre Valras et Saint-Pierre, une nouvelle station sere céée à l'embouchure de l'Aude. De là, on purra aisément gagner le parc regional de l'Espinouse et le Minervois (circuits 5 et 6 ).

4. Saint-Pierre-Gruissan. C'est le secteur le plus pittoresque de la côte languedocienne, avec le petit massif calcaire de la Clape dominat au sud les étangs et le vieux village de Gruissan, dans un paysage précis et délicat de style florentin. Le «circuit des abbayes» cisterciennes de Fontgroide, Saint-André-deRoquelongue, Saint-Hilaire... contribue à privilégier ce court secteur jusqu'ici isolé et totalement méconnu du grand tourisme international (circuits 7 et 8).

5. Leucate-Barcarès. Le site est ici identique à celui de Mamaia. La nouvelle station sera créée sur un cordon littoral de $10 \mathrm{~km}$ séparant de la mer l'étang de Leucate. Malgré les remarquables équipements des ports de plaisance, le risque est grand de se laisser entrainer à une implantation de type «social» qui vulgariserait un paysage original de vastes étangs silencieux peuplés d'une faune spécifique. Mais les Corbières proches, au potentiel touristique encore intact, et le Conflent, avec ses merveilles d'art roman catalan, réservent d'admirables découvertes (circuits 9 et 10).

6. Canet-Le Racou. Ce secteur était le plus fréquenté et le plus actif en raison de la richesse de l'arrière-pays roussillonnais. Il peut être le point de départ de belles randonnées vers la Côte Vermeille, les églises du Vallespir, les prieurés de l'Aspre (circuits 11, 12 et 13).

Sète et Frontignan garderont leurs activités industrielles et maritimes sans pour autant être exclues des préoccupations d'aménagement touristique. C'est sur les pentes du Mont-Saint-Clair et sur le lido qui sépare l'etang de Thau de la mer que seront implantés les équipements touristiques.

Sur l'ensemble du littoral, la capacité d'accueil sera portée de 255.000 à 650.000 lits (campings et toutes formes d'hébergement inclus), ce qui permettra de recevoir 2.000.000 de touristes par saison.

La réalisation de ce programme est activement menée. La démoustication est pratiquement réalisée. Les boisements progressent, non sans difficultés. Les ports de plaisance de La Grande Motte, de Leucate-Barcarès et de Saint-Cyprien sont terminés et l'equipement des trois stations est avancé ( $\mathrm{La}$ Grande Motte a accueilli dès 1967 ses premiers touristes); les chantiers sont ouverts au Cap d'Agde et à Gruissant, et le seront en 1970 a l'embouchure de l'Aude. 
L'aménagement aboutira à l'organisation d'un espace-vacances très spécialisé mais distinct du type des stations roumaines par le fait qu'il sera continu et non ponctuel, et intégrera des unités existantes: Le Grau-du-Roi, Carnon Palavas, Le Grau-d'Agde, Valras La Nouvelle, Leucate, Canet...

Il est vrai que ces très petites agglomérations sont médiocres. Leur vie propre, en dehors du tourisme, est réduite à une petite activité agricole, la pêche ayant perdu tout intérêt. Elles n'ont pas le dynamisme et la personnalité des ports de la Costa Brava. Il est probable qu'elles seront absorbées par la fonction touristique, qui dès maintenant y est prépondérante. On aboutira donc, très vraisemblablement, à une fonction unique, à un espace-vacances artificialisé, protégé, pratiquement continu sur $160 \mathrm{~km}$. Il n'evitera l'ennui que si la conception urbanistique introduit, dans ces espaces le plus souvent plats et monotones, des jeux harmonieux de volumes, d'espaces verts, de jardins, d'allées, de ports... et si on structure fortement chaque unité sur un espace central à vocation sociale affirmée, avec de vraies rues bordées de magasins et d'installations récréatives; et surtout enfin si on veille particulièrement à l'animation des stations afin de remplacer la chaleur humaine et les contacts sociaux, qui existent naturellement dans les agglomérations à activités multiples, par des activités de vacances nombreuses et diversifiées. C'est essentiellement une question d'équipement sportif et récréatif. L'attention portée aux ports de plaisance -il y en aura 12 d'un capacité totale de 16.000 places - répond à cette préocupation. La navigation introduit toujours vie et mouvement. Mais il faudra en outre tous les équipements susceptibles d'attirer une clientèle jeune. De leur attrait - et de la politique des prix- dépend le succès de l'opération.

L'aménagement du littoral du Languedoc et du Rousillon se distingue encore des créations du second type par le souci d'intégrer le littoral à l'espace régional.

L'organisation du réseau routier est conçue de manière à assurer à la fois la fluidité de la circulation sur l'axe longitudinal parallèle au rivage, et la protection de l'espace-vacances contre les nuisances liées à la grande circulation, en permettant cependant un accès facile aux stations et leur intégration dans l'espace régional.

C'est pourquoi, si l'autoroute Nîmes-Perpignan est rejetée en arrière des stations, à quelques kilomètres du rivage, des échangeurs nombreux sont prévus qui permettront d'accéder aux voies transversales dirigées vers les stations. En bordure de la mer, il n'y aura que les «routes touristiques» à circulation lente reliant entre elles les stations. La liaison avec l'arrière-pays sera assurée par l'intermédiaire des villes sublittorales: Nîmes, Montpellier, Béziers, Narbonne, Perpignan, d'où partent des nombreuses voies de pénétration. Le résultat sera un quadrillage de l'espace littoral permettant d'un part l'écoulement rapide du grand flux longitudinal et d'autre part l'établissement de flux transversaux plus lents irriguant les stations.

Ces flux comporteront:

- Des mouvements de capitaux. On constate dès à présent que les investissements sont essenctiellement d'origine locale et régionale. Chaque ville lan- 
guedocienne et rousillonnaise investit dans «sa» plage. Cependant, des acheteurs plus lointains se manifestent: parisiens évidemment, mais aussis toulousains, lyonnais, et quelques étrangers. Les Allemands avaient acquis en 1968 8.800 lits, les Anglais 7.500, les Suisses 1.180 , les Belges $400 .^{10}$

On peut craindre cependant qu'une grande partie des profits de l'opération échappe à la région. L'argent versé par l'État pour payer les terrains à aménager n'y reste pas intégralement, de nombreux propriétaires habitant ailleurs. Les travaux d'équipement ne lui assurent même pas le bénéfice de toutes les sommes investies dans la construction, car 9 entreprises adjudicataires sur 12 sont extérieures.

Dans la phase d'exploitation, la region retirera sans doute le bénéfice de la consommations des touristes (évaluée à 1 milliard de F en 1970); mais à condition que les maisons de commerce locales ne se laissent pas supplanter par les succursales d'entreprises extérieures.

Malgré ces réserves, il est certain que l'équipement entrepris a déjà déclenché des mouvements de capitaux qui contribuent à structurer l'espace littoral et à le lier à l'ensemble de l'espace régional, national et international.

- Des mouvements de main d'œuvre; mais le caractère saisonnier du tourisme posera des problèmes habituels de rupture du rythme de l'emploi, particulièrement dans les stations nouvelles.

- Des mouvements de produits assez limités et très fortement déséquilibrés à la fois dans l'espace (déséquilibre en faveur des arrivées, les stations n'ayant aucune, on seulement de très médiocres activités productrices) et dans le temps (déséquilbre saisonnier).

Quant au mouvement des touristes, il est essentiellement d'origine locale et régionale. Si l'opération réussit, l'aire d'appeel tendra à se confondre avec celle de la Côte d'Azur ou de la Costa Brava; c'est dire qu'elle s'élargira considérablement.

Mais si on veut entraîner la région dans ce mouvement d'expasion, c'est dans un rayon de 50 à $80 \mathrm{~km}$ autour de chaque station qu'il faudra développer les relations pendant la saison. Dans ce dessein, des «modèles» de circuits sont dès maintenant proposés aux touristes. ${ }^{11}$ Ils les dirigent vers un arrière-pays fortement touché par l'exode rural et demeuré jusqu'ici presque entièrement à l'écart des grands courants de relations modernes. Très mal connu, et cependant magnifique, chargé de souvenirs qui ont pris valeur de symboles, cet arrièrepays languedocien et roussillonnais pourrait devenir une des meilleures chances de l'aménagement du littoral, dans la mesure où il constitue un élément d'attraction complémentaire. ${ }^{12}$

10. Rapport au Gouvernement. Mission interministérielle pour A.T.L.R., mai 1968

11. Guide bleu Languedoc-Rousillon, Hachette, 1968, cf. supra p. 264.

12. Bien que les conditions du milieur physique et biogéographique soient profondément differentes, la côte d'Aquitaine fer l'objet d'un aménagement fondé sur les mêmes principes. Mais l'équipement y sera plus léger, plus sélectif - en raison de la fragilité du milieu biogéographique-, plus tourné vers les plans d'eau intérieurs des étangs - en raison de la longue barrière des dunes et de la violence de l'Océan. 
L’aménagement touristique du littoral du Languedoc-Rousillon aboutira à une organistation distincte à la fois:

- du type Costa Brava ou Côte d'Azur par le caractère volontaire de l'opération et par le fait qu'elle s'inscrit dans un espace totalement ou en majeure partie libre de toute occupation humaine, de sorte qu'on aboutira à la création d'un espace-vacances très spécilisé et artificialisé;

- et du type bulgare ou roumain, avec lequel il présente certes plus d'analogie, mais dont il differe par le parti pris d'aménagement global d'un espace continu et par la volonté d'intégrer l'espace littoral à la vie régionale et d'entraîner l'arrière-pays dans le mouvement d'expansion.

Ces exemples méritent d'être médités dans le cadre d'un politique d'aménagement.

Dans le premier cas étudié, le comportement économique est commandé par la demande; dans les deux autres, par l'offre.

Dans le premier cas, il importe de contrôler, et éventuellement de freiner, un équipement qui relève presque exclusivement du secteur privé.

Dans les deux autres, il s'agit de créer, ex nihilo, avec la participation totale ou essentielle des investissements publics, un équipement touristique complet. Il s'agit de savoir si les espérances de rentabilité justifient les énormes investissmenets engagés dans des actions d'aménagement d'une telle envergadure et si la formule choisi par le Languedoc peut et doit être reprise au profit de nombreux secteurs sous-exploités du litoral français dans le cadre d'un politique general d'aménagement du territoire, ou s'il ne conviendrait pas de revenir à des formules plus souples, laissant plus de champ aux mouvements spontanés et aux initiatives privées, mais dans le cadre d'une politique mieux affirmée de proteccion de la nature.

\section{Notes}

(a) Cet article, écrit fin 1969, pêchait par excès d'optimisme. Je sous-estimais les effets négatifs du tourisme. Si les principaux facteurs d'attraction touristiques que son l'eau, le soleil, le sable, le patrimoine culturel... ne sont pas, au sens littéral du mot, "consommés» par les touristes, l'espace en revanche peut être «dévoré» par l'extension galopante des équipements touristiques. Leurs effets négatifs sur l'environnement sont de plus en plus vigoureusement dénoncés, même s'il est vrai qu'en certains cas, le tourisme peut avoir l'effet inverse et valoriser l'environnement.

(b) Là encore, je me laissais emporter par ma passion pour la Costa Brava et mon amitié pour les Catalans. Malgré mes mises en garde répétées contre les excès et les dérives que ne manqueraient pas d'engendre l'explosion de la demande, la recherche incontrôlée du profit, les interventions de capitaux extérieurs... le béton a défiguré les petits ports, déferlé dans les calas, conquis des paradis jusque-là inviolés. 
Mais une réaction se dessine. Des groupes de jeunes, amoreux fous de leur littoral, s'unissent pour dénoncer les dégradations de l'environnement, qui privent le tourisme de la Costa Brava de son atout majeur, le condamnant ainsi, à terme, à la régression en terme de quantité mais surtout de qualité. Ne se contentant pas de discours, ils s'organisent, alertent les populations, entraînent les municipalités dans leur combat, bref agissent et progressent malgré la pression des intérêts contraires. Ils créent des associacions comme le groupe Demall à Lloret, les «Amis de Tossa Club unesco» à Tossa, ou la «Federació Catalana d'Associacions Club unESCO». Avec le soutien de l'unEsCo, des autorités régionales et locales (celles-ci parfois réticentes), de l'Université de Gerone, des personnalités influentes, ils ont organisé des symposiums (Lloret 1992, Tossa 1996) où son débattus des problèmes concrets, adoptés des programes d'action et des stratégies. Leur succès dépend essentiellement de la réaction et de l'adhésion des populations. Incurablement optimiste, je fais encore une fois confiance aux Catalans. 